\title{
Plurihormonal Pit-1 lineage adenoma presenting as meningitis with recurrence after somatostatin analogue
}

\section{Yoko Olmedilla1, Shoaib Khan², Victoria Young3, Robin Joseph33, Simon Cudlip4, Olaf Ansgorge5, Ashley Grossman² and Aparna Pal2}

1Endocrinology and Nutrition Service, Gregorio Marañón General Universitary Hospital, Madrid, Spain, 20xford Centre for Endocrinology, Diabetes and Metabolism, Churchill Hospital, Oxford, UK, Departments of

${ }^{3}$ Neuroradiology, ${ }^{4}$ Neurosurgery, and ${ }^{5}$ Neuropathology, John Radcliffe Hospital, Oxford, UK
Correspondence

should be addressed

to A Pal

Email

Aparna.pal@ouh.nhs.uk

\section{Summary}

A 21 year-old woman was found to have a pituitary macroadenoma following an episode of haemophilus meningitis. Biochemical TSH and GH excess was noted, although with no clear clinical correlates. She was treated with a somatostatin analogue (SSA), which restored the euthyroid state and controlled GH hypersecretion, but she re-presented with a further episode of cerebrospinal fluid (CSF) leak and recurrent meningitis. Histology following transsphenoidal adenomectomy revealed a Pit-1 lineage plurihormonal adenoma expressing GH, TSH and PRL. Such plurihormonal pituitary tumours are uncommon and even more unusual to present with spontaneous bacterial meningitis. The second episode of CSF leak and meningitis appears to have been due to SSA therapy-induced tumour shrinkage, which is not a well-described phenomenon in the literature for this type of tumour.

\section{Learning points:}

- Pit-1 lineage GH/TSH/PRL-expressing plurihormonal pituitary adenomas are uncommon. Moreover, this case is unique as the patient first presented with bacterial meningitis.

- Inmunohistochemical plurihormonality of pituitary adenomas does not necessarily correlate with biochemical and clinical features of hormonal hypersecretion.

- Given that plurihormonal Pit-1 lineage adenomas may behave more aggressively than classical pituitary adenomas, accurate pathological characterization of these tumours has an increasing prognostic relevance.

- Although unusual, a CSF leak and meningitis may be precipitated by SSA therapy of a pituitary macroadenoma via tumour shrinkage.

\section{Background}

Plurihormonal Pit-1 lineage adenomas expressing growth hormone $(\mathrm{GH})$, thyrotrophin (TSH) and prolactin (PRL) are rare $(1,2)$. Clinical expression of these plurihormonal tumours varies, as immunohistochemical staining, hormonal hypersecretion and associated clinical features may not necessarily align. Furthermore, bacterial meningitis as first presentation of a pituitary adenoma is also highly uncommon (3).
Although transsphenoidal adenomectomy is still considered the first therapeutic approach in patients with these plurihormonal adenomas (1), the role of somatostatin analogues (SSA) as primary medical therapy in selected cases appears promising $(4,5)$. In contrast to the treatment of invasive prolactinomas with dopamine agonists, therapy with SSAs is seldom associated with the development of a cerebrospinal fluid (CSF) leak and/or meningitis (6). 
We report a case of a Pit-1 lineage plurihormonal (GH/TSH/PRL) pituitary adenoma initially presenting with meningitis. On initiation of subsequent SSA therapy, this patient developed a CSF leak and a second episode of meningitis.

\section{Case presentation}

A 21-year-old woman was admitted in July 2015 with severe headache, neck stiffness and low back pain. CSF analysis confirmed this to be bacterial meningitis (Haemophilus influenzae). Imaging revealed a mass in the pituitary fossa extending inferiorly into the sphenoid sinus. The patient was treated with standard antibiotics and made a full recovery from the meningitis. On careful review of previous symptoms, the patient reported longstanding frontal headache and fatigue but no other symptoms of a functioning pituitary tumour. She had regular periods on no chemical contraception. Physical examination revealed no overt features of acromegaly, thyroid dysfunction or goitre. Visual fields were normal.

\section{Investigation}

Magnetic resonance imaging (MRI) of the sella revealed an anterior pituitary mass measuring $3 \times 2.7 \times 1.6 \mathrm{~cm}$. The tumour appeared to be centred on the right cavernous sinus with extension to the right sphenoid sinus and mild displacement of the normal pituitary gland and infundibulum to the left side. Although very close to the lesion, the optic chiasm and proximal right optic nerve were not obviously compressed. A high T2-weighted signal in keeping with fluid was apparent on the right side of the sphenoid sinus (Fig. 1).

Laboratory endocrine evaluation at presentation disclosed central hyperthyroidism in addition to an elevated serum IGF1 (Table 1). The rest of the pituitary function tests were within the normal range. Sex-hormonebinding globulin (SHBG) was 76.7 (34.0-148.0) nmol/L. Other tests to distinguish TSH hypersecretion from resistance to thyroid hormones (RTH) were not performed given emergency presentation, co-secretion of GH and subsequent decision to institute SSA.

\section{Treatment}

In view of hormone hypersecretion and the invasive nature of the pituitary tumour, it was decided to treat the patient initially with an SSA ahead of further imaging and likely surgery; lanreotide autogel $120 \mathrm{mg}$ was first
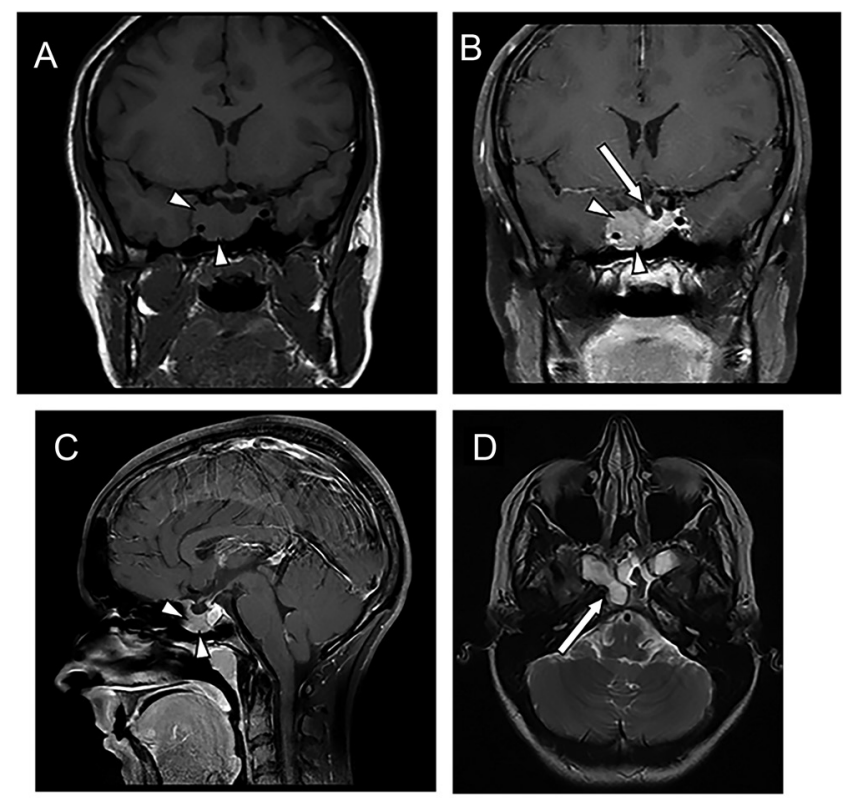

\section{Figure 1}

Magnetic resonance imaging (MRI) results at presentation, demonstrating a large pituitary macroadenoma (arrowheads in A, B and C). (A) Coronal non-enhanced T1-weighted image. (B) Coronal T1-weighted postgadolinium fat-saturated image. The normal gland is compressed by the tumour. Arrow indicates the infundibulum, which is distorted to the left and enhances more than the tumour itself. (C) Sagittal T1-weighted post-gadolinium fat-saturated image. (D) Axial T2-weighted image. Arrow indicates the high T2 signal according to fluid collected in the right sphenoid sinus.

administered almost 12 months after the presenting meningitis episode. However, 22 days following the initiation of the SSA, the patient reported a continuous drip from her nose and dull headache. Analysis of the nasal fluid confirmed CSF rhinorrhoea. The patient was admitted to hospital and contrast computed tomography (CT) scanning revealed stable appearance of the pituitary macroadenoma, with no evidence of pneumocephalus or bone destruction. Whilst an inpatient, the patient was managed conservatively and the CSF leak stopped spontaneously. SSA therapy was continued thereafter with lanreotide autogel $120 \mathrm{mg}$ every 4 weeks.

Following the second admission 1 month post initiation of the SSA therapy, the CSF leak essentially ceased with the patient reporting only very occasional episodes of 'dripping'. However, 4 months after initiating the SSA (and therefore having administered a total of four monthly doses of lanreotide autogel $120 \mathrm{mg}$ ), the CSF leak recurred and the patient presented with a new episode of bacterial meningitis, confirmed on CSF analysis. After completion of IV antibiotics, a CT cisternogram was undertaken and revealed an osseous defect in the floor of the pituitary fossa with contrast extravasation into the 
Table 1 Pituitary hormonal profile.

\begin{tabular}{l}
\hline Hormone \\
\hline ACTH (am; ng/L) \\
Cortisol (nmol/L) \\
$\quad$ SST-baseline \\
SST-30 min \\
TSH (mU/L) \\
fT3 (pmol/L) \\
fT4 (pmol/L) \\
Random GH ( $\mu \mathrm{g} / \mathrm{L})$ \\
IGF1 (nmol/L) \\
Prolactin (mU/L) \\
FSH (IU/L) \\
LH (IU/L) \\
Oestradiol \\
(pmol/L)
\end{tabular}

\begin{tabular}{|c|c|}
\hline Reference range & At presentation \\
\hline $0-46$ & 27.5 \\
\hline \multicolumn{2}{|l|}{$>430 *$} \\
\hline & 319 \\
\hline & 462 \\
\hline $0.30-4.20$ & 1.75 \\
\hline $2.6-5.7$ & 8.3 \\
\hline \multirow{2}{*}{$9.0-19.0$} & 24.7 \\
\hline & 6.7 \\
\hline $12.0-50.1$ & 56.7 \\
\hline $110-560$ & 338 \\
\hline $1-30$ & 11.0 \\
\hline $2-100$ & 18.4 \\
\hline $77-2400$ & 564 \\
\hline
\end{tabular}

\begin{tabular}{c}
\hline $\begin{array}{c}\text { Immediately } \\
\text { prior to SSA }\end{array}$ \\
\hline \\
\\
\\
\\
2.85 \\
9.8 \\
26.0 \\
19.2 \\
47.9 \\
577 \\
9.8 \\
22.6 \\
945 \\
\end{tabular}
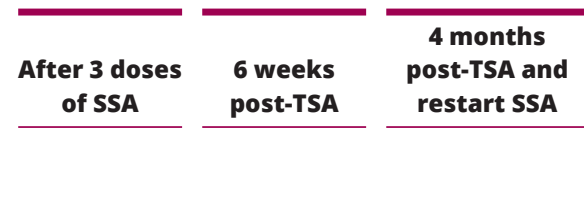

16 months

post-TSA and

12 months SSA

*Cortisol value at $30 \mathrm{~min}$

SSA, somatostatin analogue; TSA, transsphenoidal adenomectomy; ACTH, adrenocorticotropic hormone; SST, short synacthen test (at baseline and +30 min, respectively, after $250 \mu \mathrm{g}$ cosyntropin stimulation test); TSH, thyroid-stimulating hormone; fT3, free tri-iodothyronine; fT4, free thyroxine; GH, growth hormone; IGF1, insulin-like growth factor 1; FSH, follicle-stimulating hormone; LH, luteinising hormone.

right sphenoid sinus, identifying the source of the CSF leak (Fig. 2).

The patient proceeded to transsphenoidal adenomectomy and repair of the CSF leak, with no major post-operative complications. At surgery, a breach in the dura, bone and mucosa by the tumour anteriorly and inferiorly was identified as the most likely source of the CSF leak. After exploration of the pituitary fossa, the tumour was removed, and the very thin diaphragm with visible arachnoid and CSF were identified. On attempt at exploring the invaded right cavernous sinus, there was profound bradycardia, and therefore, as the tumour was rather fibrous, it was decided to avoid aggressive resection of the cavernous sinus tumour.

The elevated serum IGF1 normalised, as did the deranged thyroid function (Table 1) with SSA therapy. Normalisation of the latter in response to SSA provides supportive evidence of TSH hypersecretion in this case rather than RTH. Growth hormone day-curves (GHDCs) were performed prior to the initiation of SSA, after 3 months and 16 months post TSA, showing a decrease in hormonal hypersecretion (Table 2).

\section{Outcome and follow-up}

On light microscopy (Fig. 3), the tumour sample contained parts of a tumour composed of sheets and pseudoglandular structures of mildly pleomorphic neuroendocrine cells with round nuclei, finely granular chromatin, focally distinct nucleoli and abundant granular cytoplasm, chromophobic on PAS/OG staining. There were no vacuoles or nuclear inclusions. The tumour had the features of a pituitary adenoma, with a raised KI-67 proliferation rate (focally up to 5-10\%). Nuclear staining for p53 was weak with rare accentuation. Immunohistochemical staining showed patchy cytoplasmic expression of GH ( 30\%) with focal TSH $(\sim 5 \%)$ and PRL $(\sim 5 \%)$ expression. All tumour cell nuclei were strongly Pit-1 positive. Tumour cells did not express

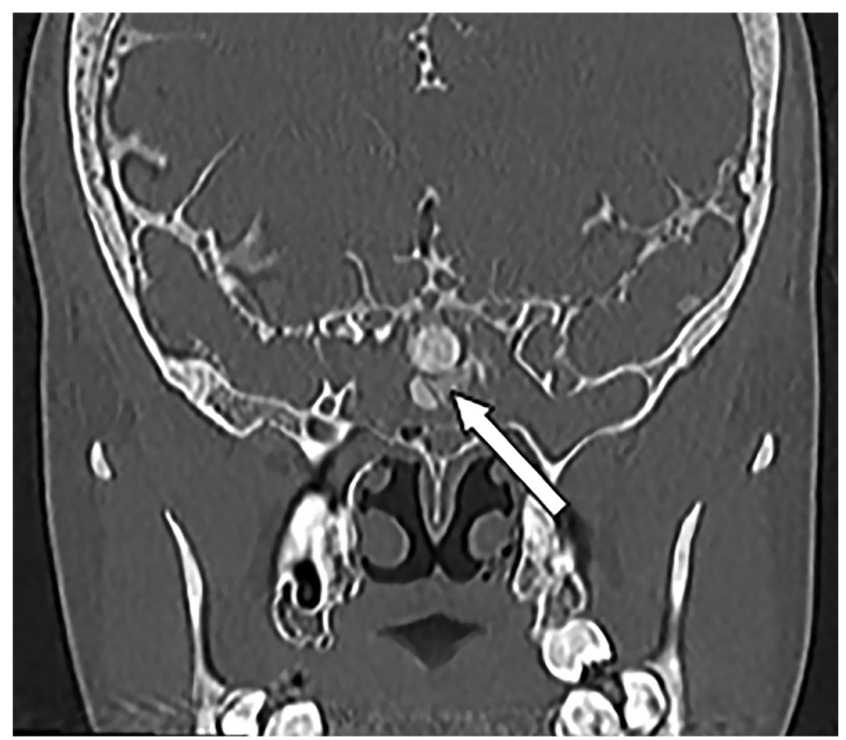

Figure 2

Computed tomography (CT) cisternogram. Arrow indicates contrast extravasation into the right sphenoid sinus. 
Table 2 Growth hormone day curve (GHDC).

\begin{tabular}{|c|c|c|c|c|}
\hline & $\begin{array}{c}\text { Basal } \\
(09: 15 \mathrm{~h})\end{array}$ & $\begin{array}{c}\text { At } 30 \text { min } \\
(09: 45 \mathrm{~h})\end{array}$ & $\begin{array}{c}\text { At } \mathbf{6 0} \text { min } \\
(10: 15 \mathrm{~h})\end{array}$ & $\begin{array}{c}\text { At } 90 \text { min } \\
(10: 45 \mathrm{~h})\end{array}$ \\
\hline Immediately prior to SSA & 19.2 & 9.5 & 7.0 & 5.9 \\
\hline After 3 doses of SSA & 4.4 & 3.3 & 2.1 & 1.6 \\
\hline Post-TSA and 12-month SSA & 1.3 & 2.6 & 6.7 & 2.8 \\
\hline
\end{tabular}

FSH, LH or ACTH. The cytokeratin pattern (Cam5.2) was diffuse (no fibrous bodies present).

SSA therapy was discontinued after surgery but restarted 4 months later in view of elevated IGF1/GH and large residual tumour volume. Subsequent IGF1 and GH levels show improvement (Tables 1 and 2). Imaging has shown slight reduction in residual tumour volume after 6 months SSA therapy.

At follow-up in April 2018, 16 months after TSA, the patient remains asymptomatic with no new CSF leak or headache. She has persisted with normalised thyroid function, IGF1 and GH levels on lanreotide autogel $120 \mathrm{mg}$ four-weekly. She will be closely followed up given the significant residual tumour and apparently aggressive nature, with the possibility of radiotherapy being required in the future.

\section{Discussion}

This case demonstrates the unusual presentation of a Pit-1 lineage plurihormonal pituitary adenoma with bacterial meningitis likely due to subclinical CSF leak,

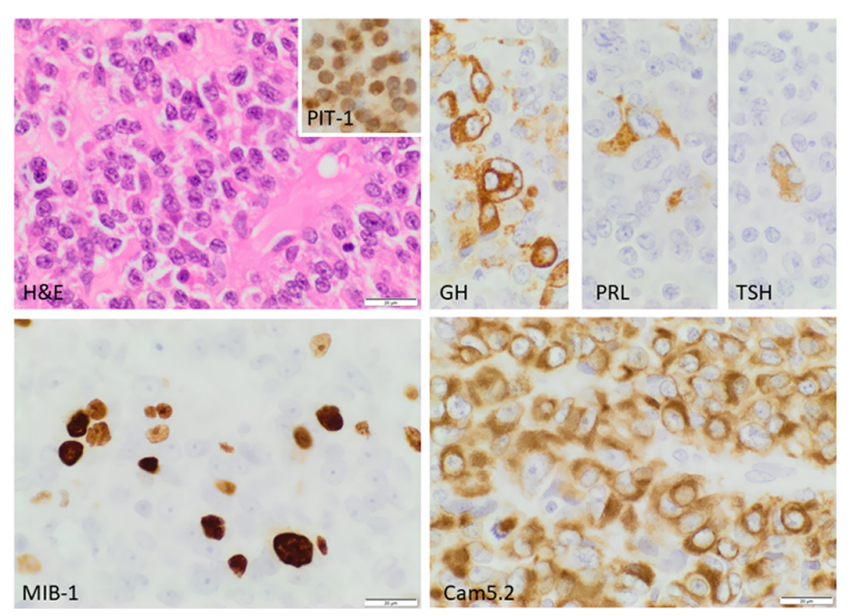

Figure 3

Neuropathology of Pit-1 lineage plurihormonal adenoma. Haematoxylin and eosin (H\&E) stain shows a mildly pleomorphic pituitary adenoma with nuclear Pit-1 expression (inset) and coexpression of growth hormone $(\mathrm{GH})$, prolactin (PRL) and thyrotrophin (TSH). The proliferation rate was focally significantly raised (MIB-1) and the Cam5.2 cytokeratin pattern was diffuse. Size bars apply to all images.

\begin{tabular}{|c|c|c|c|c|c|}
\hline $\begin{array}{c}\text { At } 120 \text { min } \\
(11: 15 \mathrm{~h}) \\
\end{array}$ & $\begin{array}{c}\text { At } 150 \text { min } \\
(11: 45 \mathrm{~h}) \\
\end{array}$ & $\begin{array}{c}\text { At } 180 \text { min } \\
(12: 15 \mathrm{~h})\end{array}$ & $\begin{array}{c}\text { At 210 min } \\
(12: 45 \mathrm{~h})\end{array}$ & $\begin{array}{c}\text { At } 240 \text { min } \\
(13: 15 \mathrm{~h})\end{array}$ & $\begin{array}{c}\text { Mean GH } \\
\text { level }(\mu \mathrm{g} / \mathrm{L})\end{array}$ \\
\hline 4.7 & 4.6 & 4.5 & 3.9 & 5.2 & 7.2 \\
\hline 1.3 & 1.2 & 1.9 & 2.8 & 2.8 & 2.1 \\
\hline 1.4 & 0.8 & 0.6 & 0.6 & 0.6 & 1.9 \\
\hline
\end{tabular}

which was subsequently exacerbated by SSA treatment. The initial CSF leak was not clinically obvious (3) but probably resulted from the tumour's extension beyond the sella turcica and subsequent bone erosion. Although CSF rhinorrhoea accompanied by meningitis is a rare first clinical manifestation of invasive prolactinomas $(6,7)$, it is not associated with presentation of other pituitary adenomas. Thus, our case is highly unusual in being a plurihormonal tumour presenting with bacterial meningitis.

Surgical cure rates are lower in patients with Pit-1 lineage plurihormonal adenomas, as these tumours are large and frequently invasive (1). Preoperative SSA therapy can induce tumour shrinkage and control hypersecretion $(4,5)$, improving general anaesthetic risk and surgical outcomes. In our case, pre-treatment with lanreotide was used to normalise thyroid function and gain tumour control following the meningitis prior to elective surgery. However, the initiation of SSA seemingly led to the unpredicted complication of a CSF leak and recurrent meningitis. The cisternogram revealed a bony defect in the pituitary fossa's floor and the adenoma may have been serving as a 'plug', preventing CSF leakage (6). Thus, tumour shrinkage post-lanreotide likely exposed the defect, leading to CSF rhinorrhoea. This effect is well established with dopamine agonist therapy of prolactinomas but not well recognised with SSAs (6).

The meningitis presentation in this case is likely a reflection of the aggressive nature of this particular tumour. The 2017 WHO classification of pituitary adenomas introduces the concept of 'Pit-1 lineage plurihormonal adenomas', which may behave more aggressively than classical adenomas, previously known as 'silent subtype 3 adenomas' (2). This is supported in our case by histology showing focally significantly raised proliferation rate, which in previous iterations of the WHO would have fallen into the 'atypical pituitary adenoma' category. Highrisk adenomas are those with rapid growth, radiological invasion, and a high KI-67 proliferation index - criteria fulfilled in our case. These factors ensure that this patient will be monitored carefully for growth and recurrence, and it is our prediction that she is likely to need radiotherapy in the future. Histological and radiological classification 
of pituitary tumours aid prediction of future behaviour; although most behave in a benign fashion, it is important to be alert to those that may behave aggressively. The Trouillas classification (8) proposes grading features for aggressiveness that are fulfilled in our case and would recognise this tumour (compatible with grade $2 \mathrm{~b}$ in this series) as one likely to recur.

The literature on Pit-1 lineage plurihormonal adenomas is scarce and the molecular mechanisms involved in thyrotroph/somatotroph adenomatous transformation have not been clearly identified. Approximately one-fourth of thyrotrophinomas are mixed adenomas, particularly the macroadenomas. These plurihormonal pituitary tumours are characterised by concomitant hypersecretion of other anterior pituitary hormones, mainly GH (16\%) and PRL (11\%) (9). This might be partly explained by overexpression of Pit-1, the pituitary specific transcriptional factor which has been found to play a role in cell proliferation in GH-, PRL- and TSH-secreting adenomas (2). Moreover, in the series of Azzalin et al., all thyrotrophinomas were found to have immunohistochemical expression of at least one pituitary hormone in addition to $\beta$-TSH, most commonly the combination of positivity for $\beta$-TSH, GH and PRL (10). Conversely, 13\% of GH-secreting pituitary tumours have been shown to demonstrate immunopositivity for TSH (11).

However, immunohistochemical plurihormonality does not always translate to hormonal hypersecretion. In the thyrotrophinomas series of Azzalin et al., preoperative oversecretion of more than one pituitary hormone occurred in only $25 \%$ of cases (10). Furthermore, such hormonal hypersecretion may not translate into clinical sequelae. In our case, although blood tests showed the tumour secreted both GH and TSH, the patient did not develop clinical features of acromegaly or thyrotoxicosis. The expected elevation in serum SHBG characteristic of thyrotoxicosis may have been attenuated by the presence of concomitant GH hypersecretion (12). Immunohistochemical analysis showed predominant expression of GH, a pattern that fitted best with the densely granulated cells observed. Despite previous serum evidence of central hyperthyroidism, the minor $(\sim 5 \%)$ TSH-positivity on biopsy may have been due to low TSH concentration in the adenomatous tissue after SSA treatment, although GH-positivity remained predominant in this context.

In summary, we report a case of a Pit-1 lineage plurihormonal pituitary adenoma presenting unusually with bacterial meningitis, most likely due to a subclinical
CSF leak. Treatment with an SSA led to an unpredicted (and rare) complication of this therapy in that tumour shrinkage caused a further CSF leak with recurrent meningitis. Our case contributes to the understanding of the clinic-pathological spectrum of the recently described Pit-1 lineage plurihormonal adenomas.

\section{Declaration of interest}

Ashley Grossman is a Senior Editor of Endocrinology, Diabetes and Metabolism Case Reports. Ashley Grossman was not involved in the review or editorial process for this paper, on which he is listed as an author. The other authors have nothing to disclose.

\section{Funding}

This research did not receive any specific grant from any funding agency in the public, commercial or not-for-profit sector.

\section{Patient consent}

Written informed consent was obtained from the patient for publication of the submitted article and accompanying images.

\section{Author contribution statement}

$Y$ Olmedilla drafted the manuscript, after a training period at OCDEM (Oxford Centre for Endocrinology, Diabetes and Metabolism), with the permission of $\mathrm{A} \mathrm{Pal}$, the physician currently responsible for the patient. $\mathrm{V}$ Young and R Joseph provided all radiological images. O Ansgorge provided all histopathological images. A Pal, A Grossman, S Cudlip and S Khan critically revised the paper. All authors have seen and approved the final manuscript for submission.

\section{References}

1 Mete O, Gomez-Hernandez K, Kucharczyk W, Ridout R, Zadeh G, Gentili F, Ezzat S \& Asa SL. Silent subtype 3 pituitary adenomas are not always silent and represent poorly differentiated monomorphous plurihormonal Pit-1 lineage adenomas. Modern Pathology 201629 131-142. (https://doi.org/10.1038/modpathol.2015.151)

2 Lopes MBS. The 2017 World Health Organization classification of tumors of the pituitary gland: a summary. Acta Neuropathologica 2017 134 521-535. (https://doi.org/10.1007/s00401-017-1769-8)

3 Robert T, Sajadi A, Uské A, Levivier M \& Bloch J. Fulminant meningoencephalitis as the first clinical sign of an invasive pituitary macroadenoma. Case Reports in Neurology 20102 133-138. (https:// doi.org/10.1159/000321844)

4 Rimareix F, Grunenwald S, Vezzosi D, Rivière LD, Bennet A \& Caron P. Primary medical treatment of thyrotropin-secreting pituitary adenomas by first-generation somatostatin analogs: a case study of seven patients. Thyroid 201525 877-882. (https://doi. org/10.1089/thy.2015.0041)

5 Amlashi FG \& Tritos NA. Thyrotropin-secreting pituitary adenomas: epidemiology, diagnosis, and management. Endocrine 201652 427-440. (https://doi.org/10.1007/s12020-016-0863-3)

6 Lam G, Mehta V \& Zada G. Spontaneous and medically induced cerebrospinal fluid leakage in the setting of pituitary adenomas: review of the literature. Neurosurgical Focus 201232 E2. (https://doi. org/10.3171/2012.4.FOCUS1268) 
7 Suliman SGI, Gurlek A, Byrne JV, Sullivan N, Thanabalasingham G, Cudlip S, Ansorge O \& Wass JAH. Nonsurgical cerebrospinal fluid rhinorrhea in invasive macroprolactinoma: incidence, radiological, and clinicopathological features. Journal of Clinical Endocrinology and Metabolism 200792 3829-3835. (https://doi.org/10.1210/jc.20070373)

8 Trouillas J, Roy P, Sturm N, Dantony E, Cortet-Rudelli C, Viennet G, Bonneville JF, Assaker R, Auger C, Brue T, et al. A new prognostic clinicopathological classification of pituitary adenomas: a multicentric case-control study of 410 patients with 8 years postoperative follow-up. Acta Neuropathologica 2013126 123-135. (https://doi.org/10.1007/s00401-013-1084-y)

9 Beck-Peccoz P, Brucker-Davis F, Persani L, Smallridge RC \& Weintraub BD. Thyrotropin-secreting pituitary tumors. Endocrine Reviews 199617 610-638. (https://doi.org/10.1210/edrv-17-6-610)
10 Azzalin A, Appin CL, Schniederjan MJ, Constantin T, Ritchie JC, Veledar E, Oyesiku NM \& Ioachimescu AG. Comprehensive evaluation of thyrotropinomas: single-center 20-year experience. Pituitary 201619 183-193. (https://doi.org/10.1007/s11102-0150697-7)

11 Mori R, Inoshita N, Takahashi-Fujigasaki J, Joki T, Nishioka H, Abe T, Fujii T \& Yamada S. Clinicopathological features of growth hormone-producing pituitary adenomas in 242 acromegaly patients: classification according to hormone production and cytokeratin distribution. ISRN Endocrinology 20132013 723432. (https://doi. org/10.1155/2013/723432)

12 Beck-Peccoz P, Lania A, Beckers A, Chatterjee K \& Wemeau JL. 2013 European Thyroid Association guidelines for the diagnosis and treatment of thyrotropin-secreting pituitary tumors. European Thyroid Journal 20132 76-82. (https://doi.org/10.1159/000351007)

Received in final form 20 January 2019

Accepted 4 March 2019 